\title{
La littérature noire et les études postcoloniales
}

David Murphy

\section{(2) OpenEdition}

Journals

Édition électronique

URL : http://journals.openedition.org/actesbranly/495

DOI : $10.4000 /$ actesbranly.495

ISSN : 2105-2735

Éditeur

Musée du quai Branly Jacques Chirac

Référence électronique

David Murphy, «La littérature noire et les études postcoloniales », Les actes de colloques du musée du quai Branly Jacques Chirac [En ligne], 3 | 2011, mis en ligne le 26 avril 2011, consulté le 07 septembre 2020. URL : http://journals.openedition.org/actesbranly/495 ; DOI : https://doi.org/10.4000/ actesbranly.495

Ce document a été généré automatiquement le 7 septembre 2020

(c) Tous droits réservés 


\title{
La littérature noire et les études postcoloniales
}

\author{
David Murphy
}

Dans L'Anti-manuel de français (1978), conçu comme un livre de lecture anarchique pour lycéens, Claude Duneton et Jean-Pierre Pagliano fournissent une critique très ironique des idées reçues de la société française de l'époque, surtout dans le domaine de l'enseignement de la langue et de la littérature françaises. Mais ils réservent une hargne particulière pour la langue de bois des hommes politiques. Dans la section du livre qui traite des méfaits de l'utilisation de la langue dans les médias, Duneton et Pagliano choisissent un extrait de l'émission de radio qui présentait en direct la finale de la Coupe de France de football en 1976. À la fin du match entre Lyon et Marseille, un journaliste demande au Président de la République de l'époque, Valéry Giscard D’Estaing, présent pour remettre le trophée aux vainqueurs, de commenter la finale gagnée par les Marseillais. Giscard, évidemment pris de court, balbutie une réponse où il se concentre sur le rôle du capitaine des Marseillais, le grand joueur Guadeloupéen Marius Trésor :

«Oui, ben je me réjouis en effet que le capitaine de l'équipe qui gagne la Coupe de France soit Trésor, c'est-à-dire un Français de Sainte-Anne, à la Guadeloupe. L'année dernière, lorsque j'ai rendu visite à la Guadeloupe, je suis allé à SainteAnne, dans la patrie de Trésor, et tous les Français sont très fiers aujourd'hui que ce soit lui qui ait conduit l'équipe de Marseille à la victoire. »

2 Dans leur " explication de texte ", Duneton et Pagliano prennent un malin plaisir à se moquer de la réponse quelque peu oblique du Président, et ils invitent leurs lecteurs lycéens à chercher la réponse à la question suivante : «Pourquoi tous les Français sontils fiers que ce soit l'Antillais Trésor qui ait conduit l'équipe de Marseille à la victoire? » Et ils donnent sept réponses possibles (il faut bien sûr " rayer les mentions inutiles ») :

1. Parce que le Président de la République française a visité la Guadeloupe ?

2. Parce que Trésor est le seul Français de l'équipe de Marseille ;

3. Parce que Trésor est le seul étranger de l'équipe de Marseille ;

4. Parce que Trésor est le plus Français des étrangers de Marseille; 
5. Parce que Trésor est le plus étranger des Français de Marseille ;

6. Parce qu'il porte un beau nom de famille ;

7. Parce que son prénom est Marius.

3 Si les pauvres lycéens arrivaient à trouver la bonne réponse, ils étaient ensuite invités à écrire une rédaction sur le sujet suivant: "Vos amis lyonnais sont venus encourager leur équipe pour la finale. Vous les rencontrez à la sortie du stade. Ils vous disent pourquoi, comme tous les Français, ils sont très fiers que ce soit Trésor qui ait conduit Marseille à la victoire. Imaginez le dialogue. »

Or, si j'ai choisi d'entamer cette communication sur la littérature noire et les études postcoloniales avec cette anecdote, c'est parce que le football, et plus généralement le sport, est avec la littérature un domaine où ont été profondément remises en question les certitudes identitaires au cours de cette dernière décennie en France. Elle nous permet de percevoir les enjeux et la complexité des débats sur le colonialisme, la race, l'identité nationale et les processus d'inclusion et d'exclusion qui existent en France : est-il possible de trouver une place pour les anciens colonisés au sein d'une reconceptualisation de l'identité française sans avoir recours à un paternalisme à la Giscard (ou bien à l'obligation d'assimiler totalement ou de quitter le territoire à la Sarkozy) ; est-il possible de renouveler notre conception de la culture française et de dépasser les idées reçues sur une France mythique? Peut-on enfin échapper à la forme symétrique, mais tellement artificielle, de l'hexagone et de son image d'une France purement métropolitaine?

5 Et la littérature noire dans tout cela? J'y arrive. De nos jours, dans le monde anglophone, la littérature dite noire, regroupant Africains, Antillais et noirs de la France métropolitaine, est rarement étudiée comme un champ de production littéraire cohérent et unifié (sauf peut-être dans des universités américaines où l'on enseigne ce qu'on appelle les «Africana Studies »); en effet, c'est souvent dans des programmes d'études postcoloniales ou de littérature comparative qu'on analyse ces textes "noirs ». Un mouvement parallèle s'est opéré dans le domaine francophone où la littérature africaine et antillaise se trouve de plus en plus intégrée dans une production francophone plus générale. De tels changements s'expliquent sans doute par un goût critique pour des catégories transnationales indépendantes de critères "raciaux » auxquels auteurs et critiques sont de plus en plus réfractaires.

Or, je dirais que la littérature noire existe toujours mais à l'intérieur d'ensembles plus larges et non fondés sur la race. Il ne faut pas négliger les différences d'approche critique entre ces " ensembles », puisque les études francophones et postcoloniales ont souvent été présentées comme antagonistes. Mais plutôt que de remuer le couteau dans la plaie créée par de vaines querelles entre critiques anglophones et francophones, je propose plutôt de traiter aujourd'hui l'une des questions les plus importantes des études postcoloniales: on parle souvent des séquelles du colonialisme dans les anciennes colonies mais la France n'a-t-elle pas été transformée elle aussi par la «mise en contact » des différentes cultures de son empire ? La question se pose donc : En quoi la France est-elle postcoloniale? S'est-il produit une remise en question fondamentale des aprioris de l'identité française depuis la décolonisation? Est-ce que la littérature noire de langue française nous aide à mieux percevoir les contours de cette France postcoloniale et les problèmes associés à son évolution? 
7 Prétendre que la France est une société postcoloniale ne signifie pas du tout que la France entière se reconnait comme telle. Comme le remarque l'un des personnages des Versets sataniques de Salman Rushdie, les Anglais ont du mal à comprendre leur histoire pour la simple raison que l'essentiel de cette histoire a eu lieu outre-mer; cette observation ne pourrait-elle pas s'appliquer aussi à la France? Je reconnais volontiers que donner une place de choix à Présence Africaine et à la littérature noire dans tout cela peut paraître chimérique. Donc si vous me permettez une dernière fois de verser dans la petite histoire, une anecdote racontée par mon collègue Thomas Spear de NYU va très bien illustrer, je l'espère, la marginalisation dont souffre une petite maison d'édition comme Présence Africaine dans le monde intellectuel parisien.

Invité sur le plateau de Bernard Pivot pour l'émission « Double je » en mai 2004, j'avais du mal à croire que cette personnalité des lettres du petit écran français ne connaissait pas la librairie Présence Africaine que j'ai choisie pour l'entretien. Cet exemple - une maison d'édition fondée à Paris il y a plus de cinquante ans - montre la place institutionnelle offerte en France à la littérature, en français, publiée en dehors de certaines grandes maisons parisiennes. Une telle ignorance de la place (dans tous les sens du terme) occupée par Présence Africaine dans les lettres de langue française paraît extraordinaire à tous ceux qui s'intéressent aux littératures dites francophones. La période coloniale a fait de la France et plus particulièrement de Paris le centre d'un vaste Empire autour duquel gravitait sa périphérie lointaine; mais, cette période coloniale a fait aussi de Paris une sorte de plaque-tournante où se réunissaient les colonisés pour parler politique, résistance et ... culture. Paris est donc devenu - et reste aujourd'hui - à travers des institutions comme Présence Africaine une porte d'entrée vers un monde " francophone » qui a été en grande partie créé par la colonisation mais qui échappe de plus en plus au contrôle d'un centre français.

Il faut qu'on s'attarde ici pour discuter plus longuement de cette question du décentrement de la langue française qui est en effet au cœur de ce débat. Il y a trois ans, Michel Le Bris, Jean Rouaud et les autres signataires du manifeste «Pour une littérature-monde en français " (dont parlera mon collègue Dominic Thomas tout à l'heure) ont souhaité voir la langue française «libérée de son pacte exclusif avec la nation», ce qui permettrait que «la langue délivrée [devienne] l'affaire de tous ». Le Manifeste cite l'exemple des écrivains de l'ancien empire britannique qui se seraient révoltés contre les normes d'une littérature « anglaise ». Et, en effet, le cas d'un Salman Rushdie ressemble sur plusieurs points à ceux d'un Tahar Ben Jelloun ou d'un Amin Maalouf.

10 Cependant, cette comparaison entre les contextes anglophone et francophone omet qu'au moment de la révolte de Rushdie et de ses pairs dans les années 1980, Londres n'était plus le centre ni des lettres anglaises ni de la langue elle-même. Aux États-Unis, au Canada, en Australie, et en Nouvelle Zélande il y a depuis très longtemps un monde de l'édition très dynamique, tandis que le Royaume-Uni lui-même est un état multinational dont la périphérie dite «celtique » a une longue tradition d'écriture en anglais.

11 Par contre, dans le contexte du monde francophone, les notions de centre et de périphérie restent de grande actualité. Les autres grandes puissances impériales européennes ont dû accepter, et cela depuis longtemps, que leurs langues ne leur appartenaient plus exclusivement: les États-Unis, l'Amérique latine et le Brésil dominent numériquement les anciens colonisateurs. Ces pays font contrepoids à la 
domination culturelle des métropoles, tandis que la France reste de loin le pays francophone le plus important dans tous les sens du terme: politique, économique, culturel, y compris dans le monde de l'édition. La France a donc mis du temps à cerner tous les enjeux du statut transnational de sa langue. Le Manifeste de Le Bris, Rouaud et compagnie a donc cherché à pousser grande ouverte la porte d'un monde des lettres de langue française transnational où il n'y aurait plus de hiérarchie entre écrivains français et francophones.

Or, cette littérature transnationale existe depuis longtemps, et l'émergence d'une littérature noire de langue française juste avant la deuxième guerre mondiale et la création de Présence Africaine juste après celle-ci sont des temps forts dans ce processus. Cette renaissance culturelle africaine et antillaise est, bien sûr, ambiguë : elle marque une fierté d'être noir qui rejette l'idée d'une assimilation totale à la culture française ; mais si cette floraison littéraire marque une sorte de rupture avec la France, elle marque aussi une profonde remise en question de l'identité française et des valeurs républicaines.

13 Je voudrais donc passer maintenant aux débuts de la littérature noire de langue française dans les années 1930. Or, si j'ai choisi de parler de Léopold Sédar Senghor dans le contexte de cette discussion d'une France postcoloniale, c'est parce que je crois qu'on a tendance à sous-estimer l'importance du défi aux valeurs républicaines que constitue la Négritude de Senghor, Césaire et Damas dans les années 1930. (Bien sûr, d'un autre point de vue on pourrait dire le contraire, à savoir que la Négritude a marqué un tournant conservateur puisque réclamer des droits au sein de la République, c'était repousser l'indépendance qui avait été réclamée par des radicaux comme Lamine Senghor dans les années 1920 ; mais il s'agit là d'un autre débat...)

14 La critique a longuement discuté, et à juste titre, de la revalorisation de la culture noire, de l'attachement de Senghor à la langue française, de ses idées sur un métissage culturel qui allait créer une Civilisation de l'Universel. Mais qu'en est-il du rapport entre la Négritude et la République? C'est Bernard Mouralis dans son excellent livre, République et colonies : entre histoire et mythe (1999) qui a poussé le plus loin la réflexion sur cette question : la Négritude est née dans cette période où la France était ce que le grand historien américain Gary Wilder appelle un "État-nation impérial »; dès lors, comment réconcilier valeurs républicaines et empire colonial?

15 Comme on le sait, la Négritude de Senghor a été sa réponse à la crise identitaire profonde qu'il a traversée dans la France des années 1930. D'abord fervent disciple d'une assimilation totale, le jeune Sénégalais a eu de plus en plus de mal à s'habituer à la culture française moderne. Le titre même de son premier recueil de poèmes Chants d'ombre (publié en 1945 bien que la plupart des poèmes datent d'avant-guerre), révèle le dilemme de l'évolué africain qui se trouve tiraillé entre deux mondes. Mais, au fur et à mesure de ses réflexions, Senghor commence à tisser une vision de complémentarité entre l'Afrique et l'Europe : par exemple, dans le poème "Prière aux masques » il proclame que le spiritualisme et le rythme africain sont « le levain qui est nécessaire à la farine blanche ».

16 On peut voir alors que, pour Senghor, réclamer une identité noire ou africaine, ce n'était pas du tout rejeter la France. En fait, il s'agissait de trouver une place au sein des valeurs républicaines pour les Noirs des colonies qui jusque-là étaient exclus de l'universalisme républicain : il faut se souvenir que la Négritude émerge à une époque où Georges Hardy, directeur de l'École Coloniale, se permet de traiter les Africains de 
«populations attardées ». Pour citer le titre de l'éditorial d'un récent hors-série du Monde sur la France d'outre-mer, la Négritude pose donc la question de la liberté, l'égalité, et de l'identité : est-ce que la fraternité de la République se limite à certains citoyens?

Or, les écrivains de la Négritude étaient loin d'être les premiers à remettre en question l'universalisme des valeurs républicaines : comme l'ont montré hier Michael Dash et comme l'aurait fait Pap Ndiaye s'il avait été là, la Révolution d'Haïti et le mouvement radical noir des années 1920 constituaient des défis majeurs. Mais pour Gary Wilder, que j'ai déjà cité tout à l'heure, l'intérêt particulier de la Négritude, c'est qu'elle émerge à un moment où se développe en Afrique française ce qu'il appelle un «humanisme colonial » qui était en train de modifier la nature du régime colonial en Afrique. Toute une génération d'administrateurs coloniaux réformateurs, tels que Maurice Delafosse, Georges Hardy et Robert Delavignette (et Senghor connaissait très bien ce dernier) s'est écartée de la vision jusque-là dominante d'une Afrique sans culture qui n'arriverait à se hisser au niveau de la culture française qu'au terme d'un long processus d'assimilation. Administrateurs, mais aussi ethnographes (amateurs), ils connaissaient et respectaient la culture des pays qu'ils gouvernaient, et dans leurs écrits, ils ont commencé à élaborer une politique coloniale non-assimilationniste, avec un système d'éducation colonial qui respecterait les valeurs et les besoins des cultures locales. Ces réformateurs coloniaux ont commencé à promouvoir une image plus positive de l'Afrique, et la Négritude a pu ajouter des voix proprement africaines à ce processus. Mais, comme l'explique Gary Wilder, si l'on pouvait à cette époque accorder une valeur positive à la culture noire, on ne pouvait pas échapper aux contradictions de la République impériale qui proposait une conception universalisante de la citoyenneté et des droits mais qui attribuait ces droits de façon très partielles dans les colonies. Le nouvel humanisme colonial tentait de créer une politique coloniale plus inclusive envers les colonisés (surtout les évolués) mais l'égalité complète vers laquelle la France prétendait amener ses sujets coloniaux était toujours repoussée dans un avenir lointain. Par conséquent, les intellectuels colonisés du mouvement de la Négritude devaient faire face à un dilemme tout à fait particulier, comme l'explique Wilder :

« Ni le républicanisme ni l'indigénisme ne constituaient une réponse efficace à un système qui prônait à la fois des politiques universalisantes et partielles. Un antiracisme qui tente d'adresser un de ces aspects du point de vue de l'autre court le risque de reproduire plutôt que de résoudre l'antinomie coloniale qu'il souhaite remettre en question. »

Pour Wilder, la Négritude des années 1930 - qui a rejeté le choix de l'assimilation mais qui a maintenu la lutte pour l'égalité - a réussi à séparer la notion de citoyenneté d'une adhésion explicite et exclusive à la culture française: pour être citoyen français et atteindre l'égalité des droits on n'était pas obligé de renoncer à sa culture d'origine. Qu'on retrouve ces mêmes questions sur la citoyenneté, la culture et l'identité dans les débats souvent controversés de la période contemporaine, deux Républiques et plus de 70 ans plus tard, souligne le radicalisme d'une Négritude qui exigeait le droit à la différence au sein de l'état-nation impérial. Et qu'on n'ait pas encore réussi à trouver une solution à ces questions souligne la naissance difficile d'une France postcoloniale.

J'arrive maintenant à ma conclusion. Dans une interview avec l'historienne Françoise Vergès, publiée en 2005, Aimé Césaire a parlé avec sa franchise habituelle des problèmes identitaires : 
«Liberté, égalité, fraternité, prônez toujours ces valeurs, mais tôt ou tard, vous verrez apparaître le problème de l'identité. Où est la fraternité ? Pourquoi ne l'a-ton jamais connue ? Précisément parce que la France n'a jamais compris le problème de l'identité.»

Au moment où l'on invite les Français à se pencher sur leur identité nationale, ce sont décidément les minorités qui sont censées poser problème. Mais, j'ai l'impression que la France devance ses hommes politiques et arrive à imaginer son identité de façon beaucoup plus complexe. Et, dans ce contexte, pour terminer, je voudrais repasser de la Martinique à la Guadeloupe, et de la littérature au football, et citer Lilian Thuram préfacier chez Présence Africaine du livre puissant de Thomté Ryam Banlieue noire - qui dit dans une interview récente :

«Je ne comprends pas ce questionnement sur l'identité nationale alors qu'elle est inscrite sur tous les frontons de mairie : Liberté, Égalité, Fraternité. Ensuite, il y a l'identité des Français, ce qui est différent. Chaque personne a sa propre identité qu'il construit chaque jour et on ne peut pas gommer ses identités dans une tentation jacobine. "

Voilà des idées qu'auraient partagées, je crois, Senghor, Césaire et Damas, ainsi que beaucoup d'autres auteurs contemporains d'une littérature noire de langue française.

\section{ANNEXES}

\section{Débat}

\section{Romuald Fonkoua}

Je voudrais remercier David Murphy pour sa contribution que je ne vais pas me permettre de résumer ici, sinon en disant simplement la chose suivante : en t'écoutant, j'avais l'impression que nous sommes passés de la V République avec Giscard, à la IV ${ }^{e}$ République avec la Négritude, Senghor et Césaire, et que nous sommes revenus ensuite à la $\mathrm{V}^{\mathrm{e}}$ République avec Thuram... Alors, ne pourrait pas dire « heureuse période de la IV ${ }^{e}$ République » ? J'ai bien l'impression, en t'écoutant, que les écrivains noirs considèrent la période où ils ont été le mieux reconnus dans la République, c'est bien sous la IV ${ }^{e}$ République, c'est-à-dire après la seconde guerre mondiale. Est-ce bien cela ?

\section{David Murphy}

La IV ${ }^{e}$ République est une période très intéressante. Elle est vraiment négligée par la critique, je pense. C'est une période où on essaye de penser à l'avenir de l'empire sous le signe de l'égalité. On essaye de réfléchir. On ne peut pas te dire qu'il y a beaucoup de mauvaise foi, mais pendant longtemps les écrivains de la négritude y croyaient. Des gens comme Senghor y croyaient, il croyait qu'il pouvait être un citoyen noir au sein de la république. La fin de l'empire ne semblait pas inévitable en 1960 pour les colonies africaines. On faisait des projections dans l'avenir, dans 20 ans en 30 ans. Je ne veux pas dire que la IV ${ }^{e}$ République, c'est mieux que l'indépendance mais c'est une période très intéressante. 


\section{Romuald Fonkoua}

Je vais passer la parole à la salle

\section{Question du public}

Pour la « farine blanche ", il faudrait que vous nous aidiez à nous situer par rapport à l'autre axiome que le président Senghor avait avancé selon laquelle l'émotion serait nègre comme la raison serait hellène. Pouvez-vous nous donner le point actuel de cette idée qui avait été avancée par le président Senghor qui à l'époque était passé inaperçue, mais a donné lieu à des polémiques très acerbes?

\section{David Murphy}

Je ne sais pas si je vais pouvoir faire le point, c'est un peu comme l'a dit hier Catherine Coquery Vidrovitch, je crois qu'il faut bien comprendre la négritude dans son contexte historique. Si on ne regarde pas la période des années 1930 et le racisme qui existe, alors on ne comprendra jamais pourquoi Senghor, Césaire et Damas veulent célébrer une identité noire qui est à l'opposé d'une identité blanche, pendant quelque temps. On a souvent dit que la négritude avait fait l'inverse de ce que disaient les colonisateurs. Donc : vous, vous chantez, vous dansez et vous n'avez pas de civilisation et ils ont dit: « oui, nous chantons, nous dansons et c'est notre civilisation ! Et vous, vous avez perdu tout cela! » Le paradoxe dans tout ça, si l'on regarde les personnages comme Senghor et c'est pareil dans les colonies britanniques - il n'était pas du genre à aller danser sur la scène, devant tout le monde. C'était un homme en costume qui faisait des discours philosophiques. Il y a un passage célèbre dans l'autobiographie de N'Krumah où il est avec un européen et regarde des gens danser en Afrique pour une fête... Il dit qu'il a honte de cette célébration du corps, du rythme, parce qu'il a peur de ce que va penser son compagnon : « mais qui sont ces gens? Ils sont fous ! « Ils dansent pour un rien ... » Il y avait donc un écart entre les " évolués ", si l'on peut utiliser ce terme, et la masse des Africains. Je crois que c'est ça, en grande partie, l'explication des idées de Senghor : il se trouve tellement loin de cette culture !

\section{Question du public}

J'ai peut-être une réflexion à ajouter sur « l'émotion des nègres et la raison hellène ». J'en ai beaucoup débattu avec lui. Je pense que l'on n'en a une vision très schématique. Moi j'ai toujours connu Senghor comme un personnage déchiré entre des aspirations qu'il n'arrivait pas à tenir et des pesanteurs dont il n'arrivait pas à se dégager. Je crois que quand il dit que l'émotion est nègre et la raison est hellène, c'est exactement les deux pôles de sa personnalité. D'ailleurs dans les dernières années de sa vie où il bascule plutôt d'un côté que de l'autre, je crois qu'il y a là quelque chose de très important. Je crois aussi qu'il ne faut pas - à ce point - assimiler le groupe de la négritude à un trio ou à un quatuor disons représentatif d'une position unique. C'est différent pour Césaire, c'est différent pour Damas, c'est différent pour Senghor. Alors, cela amène ma deuxième réflexion : je crois que cela se passe sur une autre dimension des questions que vous évoquez. Cette « conscience identitaire » a une visibilité essentiellement autour de la proclamation de la négritude, mais de façon tout aussi importante sinon plus, il y avait ce qui se passait dans les pays eux-mêmes. Là, je voudrais vraiment mettre l'accent sur le mouvement des instituteurs. À partir de l'école William Ponty et toute une série de personnages de la génération de Senghor, je pense en particulier à Mamadou Dia, à Saint-Louis du Sénégal dans ce qui s'appelait « le 
cercle de l'authenticité » ou des gens qui ne savaient pas du tout ce qui se passait en Europe. Mais eux luttaient avec une répression à la clé, alors que l'on était beaucoup plus tolérant à Paris. On se rappelle de l'une de leurs actions quand ils jetèrent ostensiblement leur casque colonial dans le fleuve au milieu du pont Faidherbe... car en tant qu'instituteurs, ils ont décidé de mettre les costumes traditionnels et furent tout de suite muté dans des postes rudes du pays. Cela permit de développer toute une réflexion et quand on prolonge tout ça on se rend compte qu'il y a eu une libération et des générateurs, il y avait des gens qui venaient de l'intérieur et il y avait des gens qui venaient de l'extérieur. Peut-être que le noyau le plus dur est venu de ces instituteurs là. Ensuite, la rupture entre Mamadou Dia et Senghor est probablement l'ultimatum le plus tragique qui se tramait à travers cet épisode de l'histoire.

\section{Romuald Fonkoua}

Merci pour cette contribution. Je ne pense pas que cela appelle une réponse.

\section{David Murphy}

Non, non, je pense que c'est très important de signaler ce qui a été fait dans l'école. Dans ma contribution, je voulais surtout parler de ce qui se passe à Paris pour montrer l'importance du projet de Présence Africaine pour la France aussi. Donc vous avez tout à fait raison et merci pour votre remarque.

\section{Romuald Fonkoua}

Le temps nous est compté. Mais si vous avez des questions, nous pourrons y revenir à la fin de la seconde communication. Merci. Merci David Murphy.

\section{AUTEUR}

\section{DAVID MURPHY}

Professeur d'études postcoloniales à l'université de Stirling (Ecosse), il est l'auteur de Sembene: Imagining Alternatives in Film and Fiction (2000) et de Postcolonial African Cinema: Ten Directors (2007). Il a aussi codirigé plusieurs recueils d'articles, dont Postcolonial Thought in the French-Speaking World (2009). 